\title{
Pigeons' memory for event duration: Intertrial interval and delay effects
}

\author{
MARCIA L. SPETCH and BENJAMIN RUSAK \\ Dalhousie University, Halifax, Nova Scotia, Canada
}

\begin{abstract}
The effects of within-session variations in the intertrial interval (ITI) and delay on pigeons' memory for event duration were studied in delayed symbolic matching-to-sample tasks. Pigeons were trained to peck one color following a long $(8 \mathrm{sec})$ sample and another color following a short $(2 \mathrm{sec}$ ) sample. In the first three experiments, the baseline conditions included a 10-sec delay (retention interval) and a 45-sec ITI. During testing, the delay was varied from 0 to $20 \mathrm{sec}$, and the ITI that preceded the trial was varied from 5 to $90 \mathrm{sec}$. When the ITI and delay were manipulated separately (Experiments 1 and 2), the pigeons displayed a choose-short tendency when the delay was longer than $10 \mathrm{sec}$ or when the ITI was longer than $45 \mathrm{sec}$, and a choose-long tendency when either the delay or the ITI was shorter than these baseline values. These effects occurred whether the sample was food access or light. When the ITI and delay were manipulated together, the pigeons showed a large choose-long error tendency when the short delay was tested together with a short ITI, and no systematic error tendency when the short delay was tested together with a longer ITI. A very large choose-short error tendency emerged on trials with a long delay and a long ITI; a reduced choose-short tendency was present when the long delay was presented together with a short ITI. In Experiment 4, the baseline conditions were a 0-sec delay and a 45-sec ITI. In this case variations in the ITI had a smaller and unidirectional effect: the pigeons showed a choose-long error tendency when the ITI was decreased, but no effect of ITI increases. Two hypotheses were proposed and discussed: (1) that pigeons judge sample durations relative to a background time composed of the I'TI and delay, and (2) that the delay and ITI effects might arise from a combination of subjective shortening and proactive effects of samples from previous trials.
\end{abstract}

Animals that have been trained to make choice responses based on the duration of a preceding event tend to make specific types of errors when the delay between the event and choice period is varied. Increases in the delay cause both rats (Church, 1980) and pigeons (e.g., Spetch \& Wilkie, 1982) to emit a disproportionate number of responses appropriate to the short event. Whether or not these choose-short errors persist over sessions depends on the delay procedure used. If the longer delays are presented only during occasional test trials, the chooseshort errors persist for many sessions (Spetch \& Wilkie, 1983). However, if the delay is fixed at the longer value for all trials of each session, pigeons appear to rapidly adjust to the new value, and the choose-short errors cease to occur within a session or two (Spetch, 1987; Spetch \& Wilkie, 1983).

Following training with a longer delay (e.g., $10 \mathrm{sec}$ ) between the signal event and choice period, the opposite type of error emerges if the delay is decreased: pigeons tend to make a disproportionate number of choose-long

This research was supported by Natural Sciences and Engineering Research Council of Canada Operating Grants U0301 (Spetch) and A0305 (Rusak). Some of these data were presented at the meeting of the Psychonomic Society in Boston, 1985. We wish to thank E. Buckle and $\mathbf{R}$. Willson for assistance with the research, and $D$. Treit and $\mathbf{R}$. Dunn for their helpful comments and suggestions. Requests for reprints should be sent to M. L. Spetch, who is now at the Department of Psychology, University of Alberta, Edmonton, Alberta T6G 2E9, Canada. errors. Spetch (1987) provided evidence that these systematic errors that pigeons display are controlled by an interaction between the training delay and the test delay. At a 5-sec test delay, pigeons made choose-short errors if the current baseline (training) delay was $0 \mathrm{sec}$, but choose-long errors if the current baseline delay was $10 \mathrm{sec}$.

These systematic errors are interesting, not only because they may provide clues about timing or memory processes (see Killeen \& Fetterman, 1988; Kraemer, Mazmanian, \& Roberts, 1985; Spetch \& Wilkie, 1983), but also because they offer an additional dependent measure with which to examine manipulations that alter overall accuracy in memory tasks. In delayed matching-to-sample (DMTS) tasks with colors as the samples, pigeons show a decrement in overall accuracy in response to both increases in the delay and decreases in the intertrial interval (ITI). Roberts and Kraemer (1982) examined pigeons' performance at various combinations of delay and ITI length, and found that accuracy decreased monotonically with increases in the delay and with decreases in the ITI. In addition, accuracy was found to be a linear function of the $\log$ of the ITI-to-delay ratio. This relationship was also found in a study using spatial locations as the sample stimuli (Wilkie, 1984). Santi (1984) found that a strong linear relationship between DMTS accuracy and the log of the ITI-to-delay ratio obtained only if the chamber was dark during the ITT. 
These observations raise some interesting questions regarding the outcome of ITI manipulations in a memory for time task. Would accuracy be affected by ITI variation when temporal events serve as the samples? Would ITI manipulations, like delay manipulations, cause the pigeons to make systematic choice errors? If so, would the direction of the errors be related to changes in the ITIto-delay ratio? Specifically, would choose-short errors occur when this ratio was decreased by either lengthening the delay or decreasing the ITI?

The present experiments explored the effects of varying the delay interval and the ITI on pigeons' overall accuracy and error patterns in delayed symbolic matchingto-sample (DSMTS) tasks with duration samples. Although Roberts and Kraemer (1982) found that effects on overall accuracy were most apparent when ITI length was varied between sessions, the present experiments examined the effects of within-session variations of the ITI and delay because of the evidence that systematic errors are more sensitive to within-session delay changes.

\section{EXPERIMENT 1}

In this experiment pigeons were trained on a DSMTS task to peck one color following a short $(2 \mathrm{sec})$ presentation of food (sample) and a different color following a long $(8 \mathrm{sec}$ ) food sample. The baseline training conditions consisted of a constant $45-\mathrm{sec}$ ITI and a constant $10-\mathrm{sec}$ delay. We examined the types of errors pigeons made on test trials with delays longer or shorter than $10 \mathrm{sec}$, and on test trials in which the preceding ITIs were longer or shorter than $45 \mathrm{sec}$.

\section{Method}

\section{Subjects}

The subjects were 5 adult White Carneaux pigeons. Birds 3-0, 3-1, and 3-2 had extensive prior training on the baseline choice task for another experiment. Birds 3-3 and 3-4 had served briefly as observers in an imitation study, but otherwise were experimentally naive prior to this experiment. Mixed grain obtained primarily during experimental sessions maintained the birds at $85 \%-90 \%$ of their free-feeding weights. The birds were housed in individual wiremesh cages; water and grit were freely available.

\section{Apparatus \\ The experimental environment consisted of Grason-Stadler animal chambers that contained three horizontally aligned response keys, each requiring a force of about $.25 \mathrm{~N}$ to operate. The keys could be transilluminated with white, red, or blue light by stimulus projec- tors mounted behind each key. The grain feeder was located below the center key, and grain presentations were accompanied by illu- mination of a lamp in the feeder. A houselight, located behind the response panel, was used only during magazine training. Experimen- tal contingencies and data recording were controlled by a DEC PDP- $8 \mathrm{e}$ computer located in an adjacent room.}

\section{Procedure}

Preliminary training. Birds 3-3 and 3-4 each received one session of magazine training, followed by a few sessions of autoshaping to establish pecking at the center key when illuminated with white light and at each of the side keys when illuminated with red or blue light. Birds 3-0, 3-1, and 3-2 required no preliminary training.

Baseline training. The baseline DSMTS training required for this experiment entailed three phases that differed only with respect to the delay interval. During all phases, the following procedure was in effect: Trials began with the presentation of white light on the center key as a trial-initiating stimulus. A single peck to this stimulus terminated it and resulted in presentation of the illuminated grain feeder as the sample. On a random half of the trials, the sample was long $(8 \mathrm{sec})$. On the remaining trials, the sample was short $(2 \mathrm{sec})$. Termination of the sample was followed by the delay interval, during which the chamber was dark, and then red and blue lights were presented on the side keys as choice stimuli. For Birds 3-3 and 3-4, blue was correct after short samples and red after long; the opposite designation was used for the other birds. The spatial position of red and blue varied across trials. A peck to either choice key terminated both stimuli; if the correct one was pecked, a 4-sec presentation of grain occurred as reinforcement. If an incorrect choice was made, the trial ended without reinforcement. A correction procedure was used during training to facilitate the learning of reference memory rules. This entailed duplicating the trial conditions on the trial that followed an error. However, accuracy was determined by performance on noncorrection trials only. Throughout training, all trials were separated by a 45 -sec ITI, during which the chamber was dark. Sessions terminated upon completion of 48 trials, or after a maximum of $52 \mathrm{~min}$.

During the first phase of training, a 0 -sec delay between sample termination and presentation of the choice stimuli was in effect on all trials. This 0 -sec delay training continued until the birds reached an accuracy criterion of at least $85 \%$ correct (overall) for 5 consecutive sessions. This required 10 sessions for Bird 3-3 and 12 sessions for Bird 3-4.

The second phase was identical to the first phase except that the delay between the sample and choice was $5 \mathrm{sec}$ on all trials. This phase lasted for 20 sessions. In the third phase, which also lasted for 20 sessions, the delay was set to $10 \mathrm{sec}$, but otherwise the procedure was unchanged.

Since Birds 3-0, 3-1, and 3-2 were already trained on the baseline procedure, they required no further training. Their training history had been identical to that described above, except that it had included a block of 20 delay testing sessions following the 0 -sec delay training. During these test sessions, 5-sec and 10-sec delays had been presented on occasional test trials interspersed among the 0 -sec delay baseline trials. Immediately prior to this experiment, these 3 birds had completed 20 baseline training sessions with a constant 10-sec delay.

Variable delay testing. The procedure used during delay testing was identical to the baseline procedure, with two exceptions. First, a correction procedure was not in effect. Second, the delay interval between the sample and choice period varied across trials in each session. The 10 -sec baseline delay was presented on a randomly determined $75 \%$ of the trials. Within each session, two test delays occurred equally often on the remaining $25 \%$ of the trials, one shorter and one longer than the 10 -sec baseline delay. On alternate sessions, these test delays were 0 and $20 \mathrm{sec}$, or 5 and $15 \mathrm{sec}$. This test phase lasted for 20 sessions.

Following this test phase, all birds were returned to the baseline training conditions (10-sec delay/45-sec ITI) for five sessions before proceding to the next test phase.

Variable FTI testing. During this test phase, the delay interval was held constant at $10 \mathrm{sec}$ on all trials, but the ITI was varied within each session. On a randomly determined $75 \%$ of the trials in each session, the preceding ITI was $45 \mathrm{sec}$; on the remaining trials, the preceding ITI was shorter or longer than this baseline value. On alternate sessions, the test trials followed ITIs of either 5 and $90 \mathrm{sec}$, or 15 and $75 \mathrm{sec}$. No correction procedure was used for this test phase, and it lasted for 20 sessions. 
Throughout both the training and the test phases, the experimental sessions were conducted 5 or 6 days a week. Sessions were conducted at approximately the same time each day for a given bird, although the times differed across birds.

\section{Data Analysis}

For the test phases of this and all subsequent experiments, the data were initially separated into five successive blocks of sessions and analyzed with blocks as a factor. However, the blocks effect was never found to be significant, and blocks did not interact significantly with any other factors in the analysis. Therefore, the data presentations were simplified by collapsing across the five test blocks. Thus, for all test phases of this and the subsequent experiments, the data reported are the averages of all test sessions conducted.

\section{Results and Discussion}

By the end of the 20 baseline sessions that preceded the test phases, all birds were performing accurately with the constant 10-sec delay. The mean accuracy of the 5 birds during the last two sessions of baseline training was $86 \%$ correct on short-sample trials, and $84 \%$ correct on longsample trials.

\section{Variable Delay Testing}

The top panels of Figure 1 show the results of the delay tests for each of the 5 subjects. The solid circles and open circles show the percentage of correct choices made on long-sample and short-sample trials, respectively. At the 10-sec baseline delay, there was no consistent difference in accuracy between short- and long-sample trials. However, at delays longer than $10 \mathrm{sec}$, there was a systematic tendency to make choose-short errors (i.e., most of the errors entailed incorrect short choices as reflected by the lower accuracy on long-sample trials). At the delays shorter than $10 \mathrm{sec}$, choose-long errors predominated. A two-way repeated measures analysis of variance revealed a significant main effect of delay $[F(4,16)=$ $18.4, p<.001 \mathrm{~J}$. The effect of sample duration just failed to reach significance $[F(1,4)=7.6, p>.05]$, but there was a highly significant interaction between delay and sample duration $[F(4,16)=25.4, p<.001]$.

These results provide a direct replication of those reported previously (Spetch, 1987). Discrepancies between the test delay and the baseline delay cause pigeons to display orderly error tendencies in these tasks.

\section{Variable ITI Testing}

The results of the ITI manipulations are shown in the bottom panels of Figure 1. The accuracy scores for each of the 5 subjects are shown as a function of the duration of the ITI that preceded each trial. A very similar pattern of results to that observed during delay manipulations is apparent: Choose-short errors predominated on trials preceded by an ITI that was longer than the 45 -sec baseline value, whereas choose-long errors occurred more often when the preceding ITI was shorter than $45 \mathrm{sec}$. Again, no consistent error tendencies were apparent on trials that followed the baseline 45-sec ITI. The analysis of variance revealed a significant effect of ITI $[F(4,16)=$ $6.0, p<.01]$ and of sample duration $[F(1,4)=9.2$, $p<.05]$. The interaction between ITI and sample duration was highly significant $[F(4,16)=46.2, p<.001]$.

Apparently, discrepancies between the current ITI and the baseline training ITI also caused pigeons to make systematic errors of both types. The similarity between the delay and the ITI functions is quite striking: In both cases, test values that exceeded the baseline value produced a tendency to make short choices, whereas test values less than the training value produced a choose-long tendency. However, this result is precisely opposite that expected if the errors arose from changes in the ITI-to-delay ratio: Increases in the ITI and increases in the delay change this

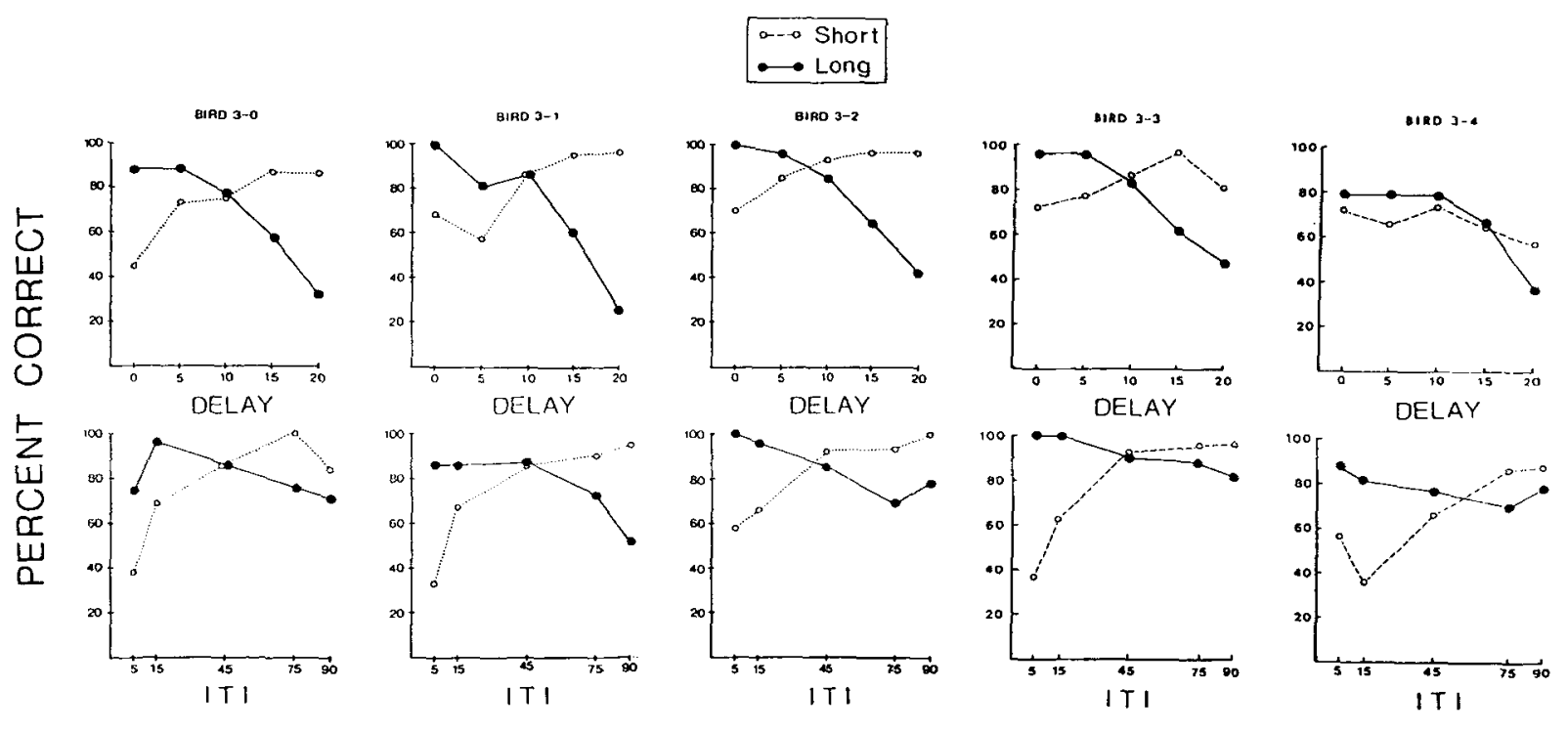

Figure 1. Percentage of correct choices on short-sample and long-sample trials as a function of the duration (in sec) of delay (top panels) and the duration (in sec) of the preceding intertrial interval (ITi; bottom panels) for the 5 birds tested in Experiment 1 . 
ratio in opposite directions. Therefore, if errors were controlled by this ratio, the delay and ITI functions should be opposite to each other, rather than qualitatively similar.

The systematic errors that occurred in response to ITI variation may, however, be consistent with some recent results obtained by Fetterman, Killeen, and Evans (cited in Killeen \& Fetterman, 1988) using a temporal scaling procedure (e.g., Stubbs, 1976). They found that pigeons' psychometric timing functions shifted temporarily following changes in the ITI. The direction of the shifts they observed seems consistent with the types of errors pigeons displayed in the present experiment. For example, following a decrease in the ITI, their pigeons switched to the "long" stimulus earlier in the trial; following an increase in the ITI, they switched to long later in the trial.

\section{Effects of Delay and ITI Variations on Overall Accuracy}

Figure 2 shows the mean overall accuracy scores as a function of delay (top panel) and ITI (bottom panel). In both cases, the highest accuracy score occurred on the baseline trials (10-sec delay/45-sec ITI). However, there is some asymmetry in that the largest decrements in over-

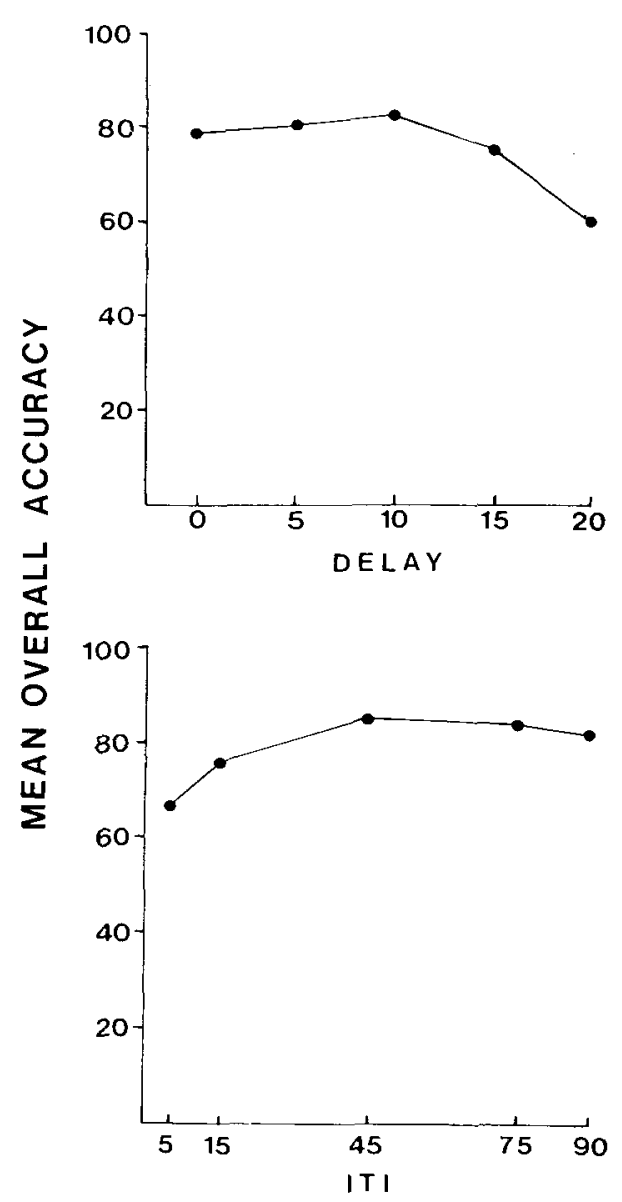

Figure 2. Mean overall accuracy as a function of delay (top) and intertrial interval (ITI; bottom) for the 5 birds in Experiment 1. all accuracy were produced by increases in the delay, but by decreases in the ITI. The direction of this asymmetry is consistent with the typical effects of delay and ITI on overall accuracy when presentations of colors or forms are the sample events.

\section{EXPERIMENT 2}

The apparent similarity between the effect of delay and ITI variation on systematic errors was intriguing and deserved further attention. Experiment 2 sought to provide a direct replication of the results obtained in Experiment 1 using another group of pigeons, both to establish the reliability of the effects and to provide a larger data base with which to assess the degree of similarity between the delay and ITI functions. In addition, this experiment examined the generality of the delay and ITI effects to a task involving light duration instead of food duration as the sample event. On the basis of previous results (Spetch \& Wilkie, 1983), we expected that the delay effects would replicate when light was used as the sample, but we did not know whether the ITI effects would also prove to generalize to sample events other than food.

\section{Method}

Subjects

The subjects were 11 adult White King pigeons. Six of the pigeons (food group) had been trained on the baseline task as part of another experiment. The remaining birds (light group) had served briefly in an autoshaping experiment, but otherwise were experimentally naive. Birds in the food group were maintained at $85 \%-90 \%$ of their free-feeding weight, whereas birds in the light group were maintained at $80 \%-85 \%$ weight. Housing conditions were as described in Experiment 1.

\begin{abstract}
Apparatus
Birds in the food group were tested in the apparatus described in Experiment 1. The experimental apparatus for the light group was a BRS/LVE chamber that was similar to that used in Experiment 1 except that it contained only two response keys, which were transilluminated with red or green light. The grain feeder was centered below and between the two keys, and a 4.8-W houselight was located at the top of the intelligence panel, directly above the feeder.

\section{Procedure}

Preliminary training. Birds in the light group received one session of magazine training, followed by a few sessions of autoshaping to establish reliable pecking to red and green illuminations of each key. The food group required no preliminary training.

Baseline training. Birds in the food group were already trained on a 10-sec delay/45-sec ITI baseline procedure identical to that described in Experiment 1. Three birds had red correct for short and blue for long, and the other 3 had the opposite arrangement. The training histories of all birds were the same as those described for Birds 3-0, 3-1, and 3-2 in Experiment 1.

The baseline procedure for birds in the light group differed only in the following ways. First, there was no trial-initiating stimulus. Second, the sample event consisted of illumination of the houselight. Third, the duration of the long sample was $10 \mathrm{sec}$ rather than $8 \mathrm{sec}$. Fourth, the choice stimuli were red and green lights; for 3 birds red was correct for short and green for long, whereas the opposite arrangement was used for the other 2 birds. The baseline training given to these birds consisted of the same three phases described in Experiment 1 (i.e., 0-sec delay training to an accuracy
\end{abstract}


criterion, 20 sessions of $5-\mathrm{sec}$ delay training, and 20 sessions of 10 -sec delay training). Throughout training the ITI was constant at $45 \mathrm{sec}$.

Variable delay and ITI testing. For both groups, the delay and ITI manipulations were carried out in an identical manner to that described in Experiment 1, with the exception that the longest test ITI was $85 \mathrm{sec}$ instead of $90 \mathrm{sec}$

\section{Results and Discussion}

\section{Variable Delay Testing}

The top left panel of Figure 3 shows the results for the food group, and the top right panel shows the results for the light group, in terms of the percentage of correct choices on short-sample and long-sample trials as a function of delay length. Consistent with expectations based on previous research, pigeons in both groups showed a tendency to make choose-short errors at delays longer than the baseline value and choose-long errors at delays shorter than baseline.

These results were analyzed separately for each group using two-way analyses of variance. The food group showed a significant main effect of delay $[F(4,20)=$ $18.45, p<.001]$ and of sample duration $[F(1,5)=7.59$, $p<.05]$, as well as a significant delay $\times$ sample duration interaction $[F(4,20)=26.7, p<.001]$. For the light group, the main effect of delay just failed to reach significance $[F(4,16)=2.84, p>.05]$, and there was no significant effect of sample duration $[F(1,4)=.29$, $p>.1]$, but the interaction between delay and sample duration was significant $[F(4,16)=3.69, p<.05]$.

\section{Variable ITI Testing}

The bottom panels of Figure 3 show the percentage of correct short and long choices as a function of ITI length
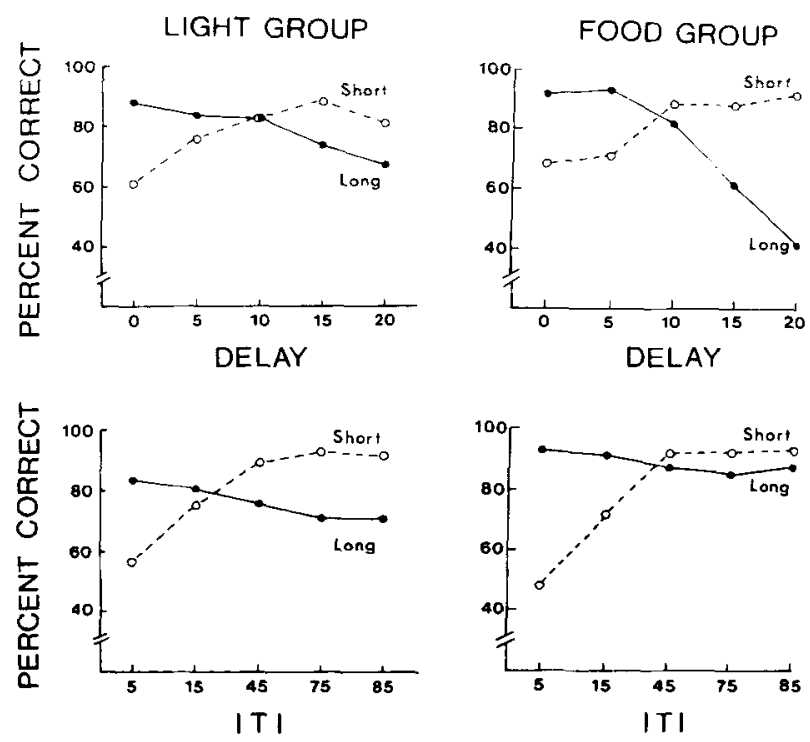

Figure 3. Mean percentage of correct choices on short-sample and long-sample trials as a function of delay (top panels) and intertrial interval (ITI; bottom panels) for birds that had light as the sample (left panels) and birds that had food as the sample (right panels) in Experiment 2.
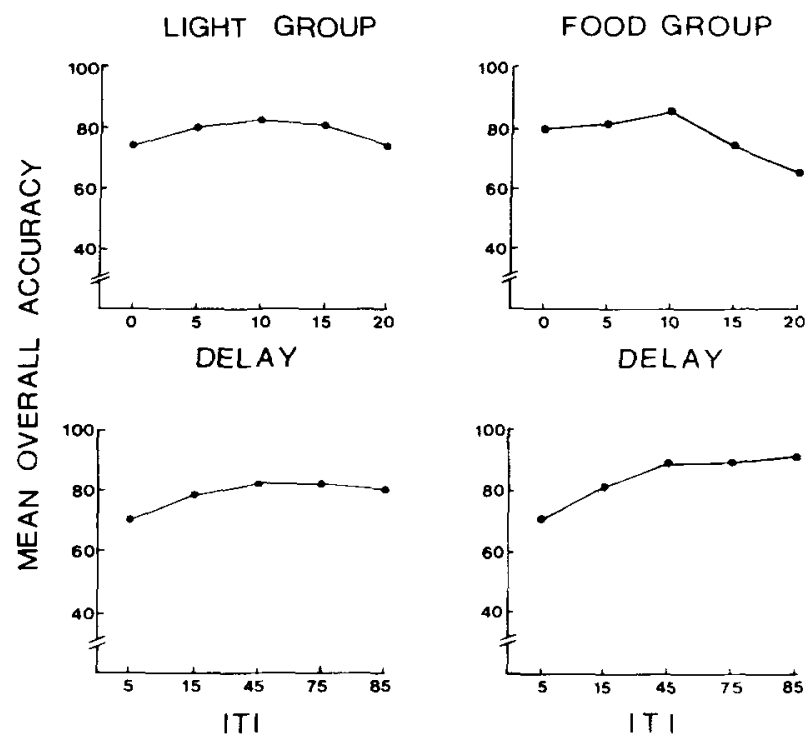

Figure 4. Mean overall accuracy as a function of delay (top) and intertrial interval (ITI; bottom) for birds with light as the sample (left) or food as the sample (right) in Experiment 2.

for both groups. As in Experiment 1, pigeons made more choose-long errors on trials that followed ITIs shorter than the 45-sec baseline value, and more choose-short errors on trials that followed longer ITIs. Although this general pattern is present for both groups, the choose-short tendency on long ITI trials was weak for the food group.

Analyses of variance on the results for the food group revealed significant main effects of both ITI $[F(4,20)=$ $10.01, p<.001]$ and sample duration $[F(1,5)=13.82$, $p<.05]$, as well as a significant interaction between ITI and sample duration $[F(4,20)=29.49, p<.001]$. The light group showed a significant main effect of ITI $[F(4,16)=4.22, p<.05]$, but not of sample duration $[F(1,4)=.57, p>.1]$, and a highly significant interaction between ITI and sample duration $[F(4,16)=17.08$, $p<.001]$.

\section{Effects of Delay and ITI on Overall Accuracy}

Figure 4 shows the mean overall accuracy of the food group (left) and the light group (right) as a function of delay length (top panels) and ITI length (bottom panels). The asymmetry between the effect of delay and ITI variations on overall accuracy is again apparent for the food group: Accuracy decreased most dramatically on trials with longer delays and shorter ITIs. The light group showed a similar pattern on ITI tests, but did not show the asymmetry during delay tests. The main effect of delay was not quite significant for this group.

The results of this experiment replicate those of Experiment 1 in revealing that within-session variations in the ITI produce systematic errors quite similar to those produced by analogous variations in the delay interval. Overall accuracy levels were also affected by variation in both ITI and delay. However, accuracy once again was found to be more disrupted by decreases in the ITI, 
whereas it was generally more disrupted by increases in the delay.

The fact that birds trained with light as the sample behaved similarly to those trained with food as the sample extends the generality of these results, and also demonstrates that the ITI effects are not dependent on the use of food as both the sample and the reinforcer.

\section{EXPERIMENT 3}

The similarity of the ITI and delay effects is suggestive of a common mechanism. To the extent that this is true, it might be possible to eliminate or at least reduce the errors normally shown at a given test delay by varying the ITI in the opposite direction; that is, the chooseshort bias seen at long test delays might be counteracted by a short ITI, and the choose-long effect observed with short delays might be eliminated by presenting a long ITI.

In the first two experiments, ITI and delay length were varied during separate experimental phases. The present experiment examined effects of combined delay and ITI manipulations. Accordingly, on some test trials, the delay and the ITI were either both shorter or both longer than the baseline value. On other test trials, the shorter ITI was combined with the longer delay and vice versa. Of particular interest was the extent to which the systematic errors produced by ITI and delay variations would cancel each other, and whether overall accuracy would improve if cancellation of the systematic errors did occur.

\section{Method}

\section{Subjects and Apparatus}

The 5 birds from Experiment 1 served as the first set of subjects. At a later time, 4 of the subjects from the food group of Experiment 2 also were tested in this experiment. The apparatus was the same as that described in Experiment 1.

\section{Procedure}

Subjects served in this experiment directly after they completed Experiment 1 or 2 . Two types of test sessions were each in effect for 10 sessions in an alternating order. During both types of tests, $75 \%$ of the trials were baseline trials (45-sec ITI/10-sec delay), and the remaining $25 \%$ were test trials. During opposition tests, half of the test trials contained a shorter delay $(0 \mathrm{sec})$ together with a longer ITI $(90 \mathrm{sec})$, and half contained a longer delay $(20 \mathrm{sec})$ together with a shorter ITI $(5 \mathrm{sec})$. During combination tests, half of the test trials contained the shorter delay together with the shorter ITI (0-sec delay/5-sec ITI), and half contained the longer delay and longer ITI (20-sec delay/90-sec ITI).

\section{Results and Discussion}

Accuracy on the baseline trials was high (mean of the 9 birds was $88 \%$ correct for short samples, $89 \%$ for long) and did not differ significantly during the two types of test sessions (88\% overall on opposition tests, $89 \%$ on combination tests). Figure 5 shows the mean percentage of correct choices for short and long samples during the test trials for the two sets of birds tested. The results are shown in terms of performance at each delay as a function of ITI. In the 0-sec delay trials (left panels), there was a clear choose-long effect at the 5-sec ITI that was completely eliminated at the 90-sec ITI. Thus, the chooselong errors that typically occur on short-delay test trials seemed to be completely countered by the longer ITI. With the 20-sec test delay (right panels), the choose-short tendency was strongly reinforced by the use of a 90-sec ITI; it was reduced but not eliminated with the 5-sec ITI.

An analysis of variance on the data from these four test conditions revealed significant main effects of delay $[F(1,8)=34.14, p<.001]$ and of ITI $[F(1,8)=30.87$, $p<.001]$. In addition, there were significant two-way interactions between delay and ITI $[F(1,8)=13.42$, $p<.001]$, delay and sample duration $[F(1,8)=91.2$, $p<.001]$, and the ITI and sample duration $[F(1,8)=$ $65.41, p<.001]$. However, the three-way interaction between delay, ITI, and sample duration failed to reach significance $[F(1,8)=2.87, p>.1]$.

Thus, variations in the ITI partially countered the effects of delay variation on systematic errors. In particular, the short test delay and long ITI appeared to completely neutralize each other when combined: under this condition the birds performed very accurately with no systematic error tendency. On the other hand, the short ITI only partly counteracted the effect of the long delay. Furthermore, as evident in Figure 6, the reduction in a choose-short bias that did occur when a 5-sec ITI was used with the 20-sec delay was not accompanied by an improvement in overall accuracy. Instead, the birds made approx-

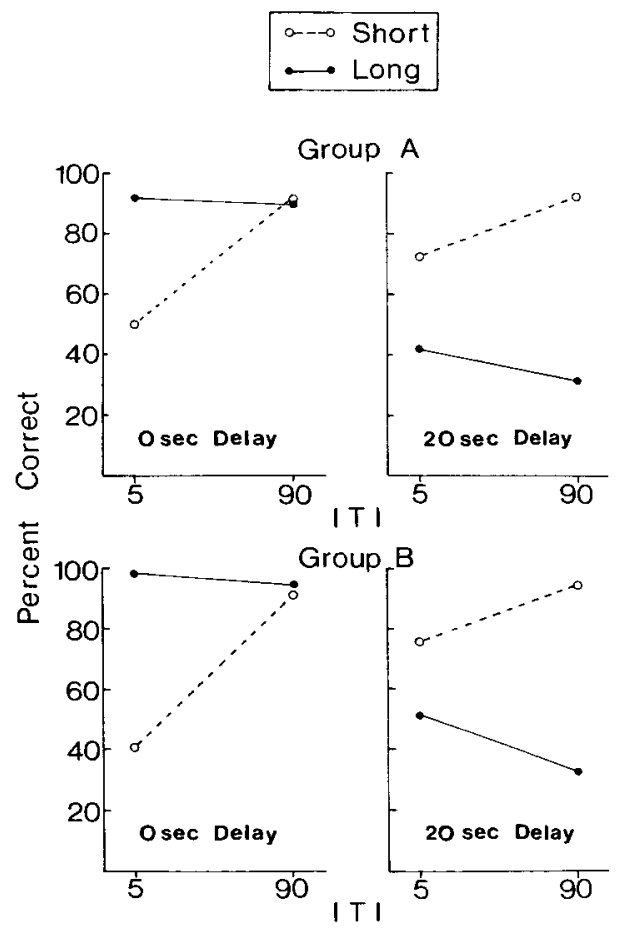

Figure 5. Mean percentage of correct choices on short-sample and long-sample trials at each of the four combinations of test delay and test intertrial interval (IT) in Experiment 3. The top panel shows the results for the first set of birds tested, and the bottom panel for the second set of birds. 


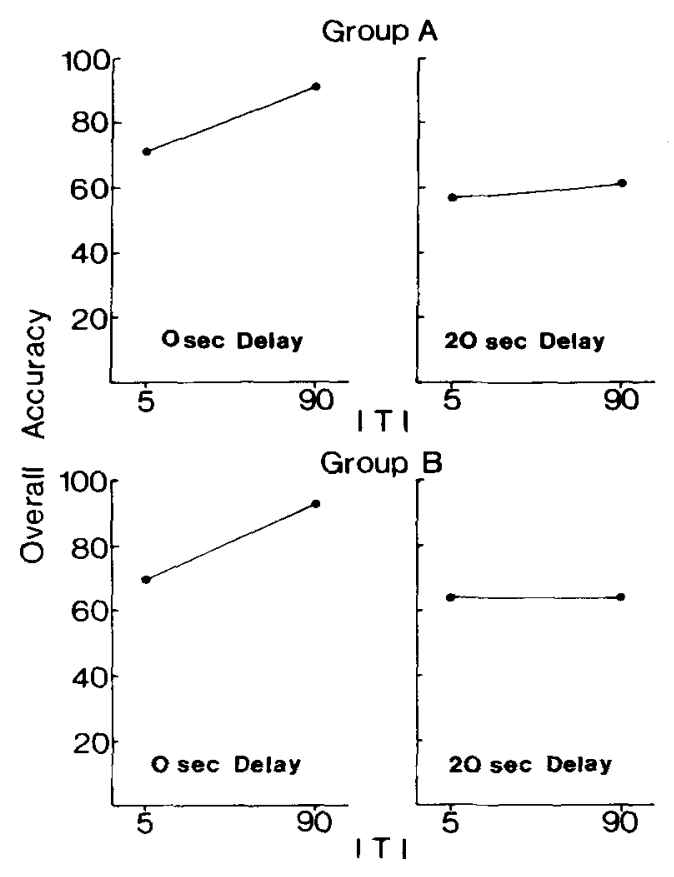

Figure 6. Mean overall accuracy at each of the four combinations of test delay and test intertrial interval (IT) in Experiment 3. The top panel shows the results for the first set of birds tested, and the bottom panel for the second set of birds.

imately the same number of errors in this condition as they did in the $20-\mathrm{sec}$ delay/90-sec ITI condition, but the errors were less systematic.

\section{EXPERIMENT 4}

Thus far, all ITI manipulations were conducted in the context of a rather lengthy $(10 \mathrm{sec})$ baseline delay. This experiment examined whether varying the ITI would generate the same pattern of systematic errors if the baseline delay were $0 \mathrm{sec}$ rather than $10 \mathrm{sec}$.

\section{Method \\ Subjects and Apparatus \\ The subjects were 5 experimentally naive White King pigeons. They were maintained at $85 \%-90 \%$ of their free-feeding weights, and housed as described in Experiment 1. The experimental ap- paratus was the same as that described for the light group of Ex- periment 2 .}

\section{Procedure}

Preliminary training. All birds received one or two sessions of magazine training, followed by a few autoshaping sessions to establish pecking to red or green illuminations of each key.

Baseline training. The baseline procedure was identical to that described in Experiment 1 except that there was no initiating stimulus; trials began directly with 2 - or 8 -sec food presentations as the short and long samples. The comparison stimuli were red and green lights. For 3 birds, red was correct for short and green for long; the opposite arrangement was used for the others. All birds were trained with no delay between the sample and choice period and a constant 45 -sec ITI until they attained an overall accuracy criterion of $85 \%$ correct for five consecutive sessions.
Delay and ITI testing. During this phase each bird first received 10 sessions in which the ITI was varied. The $45-\mathrm{sec}$ baseline ITI preceded $50 \%$ of the trials in each session, whereas 5-sec and 90 sec ITIs each preceded $25 \%$ of the trials. Following ITI testing, each bird then received 10 sessions of delay tests in which the 0sec delay occurred on half of the trials, and delays of 5 and $10 \mathrm{sec}$ occurred equally often on the remaining trials.

\section{Results and Discussion}

Figure 7 shows the mean accuracy of the 5 birds on short-sample and long-sample trials as a function of ITI (top panel) and delay (bottom panel). Variation of the ITI had a smaller effect on error tendencies in this experiment than in the previous ones. Furthermore, an effect was seen only in one direction: Decreases in the ITI produced a choose-long error tendency, but increases in the ITI had no apparent effect. Nevertheless, the analysis of variance revealed a significant main effect of ITI $[F(2,8)=21.43, p<.001]$ and of sample duration $[F(1,4)=9.73, p<.05]$, as well as a significant ITI

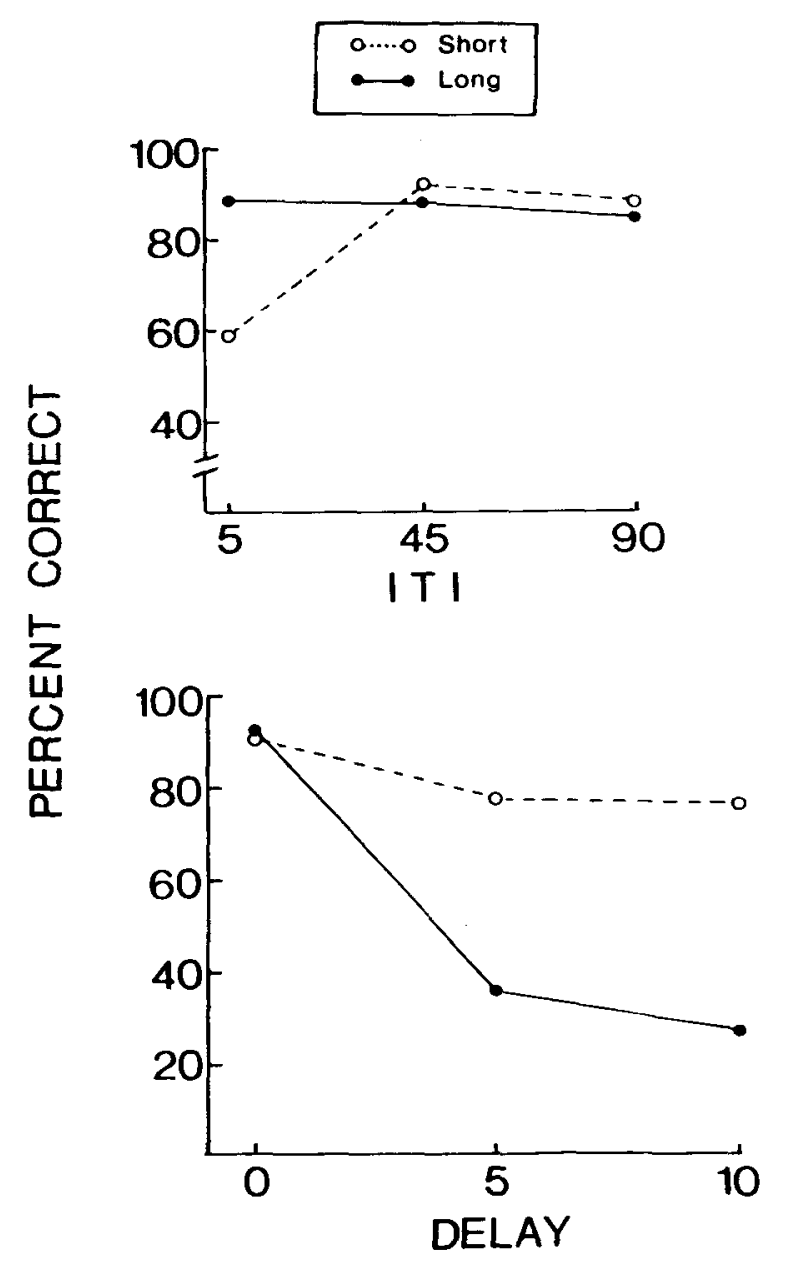

Figure 7. Mean percentage of correct choices on short-sample and long-sample trials as a function of intertrial interval (ITT; top panels) and delay (bottom panels) for birds that had a 0-sec baseline delay in Experiment 4. 


\author{
$\times$ sample duration interaction $[F(2,8)=23.71$, \\ $p<.001]$.
}

In contrast to the rather small effects of ITI variation, increases in the delay produced a large choose-short tendency, as has been found in many previous experiments. The analysis of variance for these data revealed significant effects of delay $[F(2,8)=73.88, p<.001]$ and sample duration $[F(1,4)=8.99, p<.05]$, as well as a significant interaction between delay and sample duration $[F(2,8)=11.9, p<.005]$.

\section{GENERAL DISCUSSION}

Pigeons' choice behavior in delayed symbolic matchingto-sample tasks with temporal samples is clearly sensitive to variations in the preceding ITI. Pigeons trained with a constant 45 -sec ITI consistently displayed chooselong errors on test trials that were preceded by ITIs shorter than this baseline value. Conversely, on test trials that were preceded by an ITI longer than this baseline value, pigeons were more likely to display a choose-short error tendency. These systematic errors are remarkably similar to those that emerge in response to variations in the delay interval: Delays shorter than the baseline value produce choose-long errors, whereas delays longer than the baseline value result in choose-short errors. The similarity of the two effects suggests a common underlying mechanism.

Although this common mechanism remains unclear, it does not appear to be related to the ITI-to-delay ratio, which is often correlated with overall accuracy when nontemporal events are used as samples (e.g., Roberts \& Kraemer, 1982; Wilkie, 1984). This ratio becomes larger with increases in the ITI and smaller with increases in the delay, yet pigeons in the present experiments displayed the same type of errors in both cases.

Two alternative hypotheses for the present set of results seem worth considering. One, which we refer to as the relative-duration hypothesis, is that the pigeons judge the duration of the sample not in terms of its absolute value, but instead relative to the "background" time that surrounded it, namely the ITI plus the delay. The ratio of sample-to-background time would be larger for long samples than for short samples and could form the basis of the discrimination.

Although computation of sample-to-background ratios and their subsequent comparison seems to be a cumbersome way of solving a duration discrimination task, there is evidence suggesting that pigeons can perform each of the steps that may be required. For example, pigeons can make comparisons between the durations of successively presented red and green samples for a less-than/greaterthan choice (Fetterman \& Dreyfus, 1986). They also seem able to sum over separate presentations of these samples to respond on the basis of total red and green durations (Stubbs, Dreyfus, \& Fetterman, 1984). Pigeons can even choose according to whether one duration exceeds another by a given criterion ratio (e.g., that a red stimulus was more or less than twice the duration of green; Fetterman,
Stubbs, Dreyfus, Regan, \& Bernstein, 1985). Moreover, there is evidence that rats can simultaneously time both ITI and sample duration (Meck \& Church, 1982). On the basis of these results, it is reasonable to assume that pigeons could make comparisons between ratios of sample-to-background durations.

If the temporal discriminations were based on sampleto-background ratios, then choose-short errors would be expected whenever the delay or the ITI were increased because both of these manipulations would decrease the sample-to-background ratio. Conversely, decreases in either the delay or ITI would increase the ratio and should produce choose-long errors. With an additional assumption that recent time intervals (i.e., the delay) weigh more heavily than remote intervals (i.e., the ITI), this relativeduration hypothesis seems able to account for many of the trends observed in these experiments.

Our second hypothesis, which we refer to as the temporal-summation hypothesis, is that the ITI and delay effects arise from a combination of proactive interference and subjective shortening. Spetch and Wilkie (1983) proposed that event durations become subjectively shorter in working memory as they become more remote in time, and that these changes generate the systematic errors that arise from delay manipulations. Recently, Wilkie (1988) suggested that subjective shortening may also be the mechanism whereby temporal information is normally cleared from memory in a gradual fashion after a trial is over. He found that the sample duration of a trial influences responding on the next trial when short ITIs are used, suggesting that pigeons' memory for temporal information is not simply reset at the end of a trial. Furthermore, in a recent experiment that employed an intratrial proactive interference design, samples presented several seconds before the target sample appeared to increase the subjective duration of the target sample (Spetch \& Sinha, 1988).

These notions can be integrated with the present set of results as follows. Consider that the subjective duration of a sample at the time of choice is influenced not only by the most recently presented sample, but also by samples presented on previous trials. Assume further that this perceived sample duration is the sum of all remembered sample durations (an aggregate sample). Finally, assume that all sample durations undergo subjective shortening over time. The longer the ITI, the less influence exerted by previous samples; hence, the shorter the aggregate sample seems. The longer the delay, the more subjective shortening will have occurred; hence, the shorter the aggregate sample seems. Thus, according to the temporalsummation hypothesis, a combination of subjective shortening and summation across samples from preceding trials could produce the general pattern of systematic errors observed in the present experiments.

Both the relative-duration hypothesis and the temporalsummation hypothesis may be quantified by elaborating upon a model of event memory recently proposed by Staddon (1984). Staddon's model assumes that events become progressively less salient as they recede into the past. 
Since an event's saliency is assumed to be directly related to its duration and inversely to its age, the change in saliency over a delay within Staddon's model is analogous to the subjective shortening process proposed iy Spetch and Wilkie (1983). However, Staddon's model has the advantage of offering a mathematical discription of this process. Specifically, the saliency $(S)$ of a particular event is defined by

$$
S=A\left(t_{1}^{m}-t_{2}^{m}\right),
$$

where $t_{1}$ is time since the event ended, $t_{2}$ is the time since it began, $A$ is a constant of proportionality, and $m$ represents the rapidity with which saliency declines with age $(m<0)$.

This equation can be modified to include the assumptions of each of the hypotheses we have proposed. For the relative-duration hypothesis, we modified and expanded Staddon's equation to allow for comparisons of relative event saliencies. Specifically, the relative saliency of each sample was assumed to be the ratio of sample saliency to background saliency (ITI + delay). Thus,

$$
S=\left(t_{2}^{m}-t_{1}^{m}\right)_{s} /\left[\left(t_{2}^{m}-t_{1}^{m}\right)_{d}+\left(t_{2}^{m}-t_{1}^{m}\right)_{i}\right],
$$

where $s=$ the current sample, $d=$ the current delay, and $i=$ the ITI that preceded the trial. As in Staddon's equation, $t_{1}$ is the time since the event ended and $t_{2}$ is the time since it began. However, for our equations, we reversed these and assumed that $m<1$.

For the temporal-summation hypothesis, we modified Staddon's equation to allow for summation across samples from preceding trials; that is, the saliency of the current sample was assumed to reflect the sum of all samples presented, with the stipulation that the saliency of each sample diminished with age. Thus, the saliency of the aggregate sample on trial $n$ would be described by

$$
S=\left(t_{2}^{m}-t_{1}^{m}\right)_{n}+\left(t_{2}^{m}-t_{1}^{m}\right)_{n-1}+\ldots\left(t_{2}^{m}-t_{1}^{m}\right)_{n-n} .
$$

Again, $t_{1}$ is the time since the event ended, $t_{2}$ is the time since the event began, and $m<1$.

We compared the fit of both of these equations to the present set of results, with $m$ set to .5 . The results of these calculations are shown in the Appendix. For both hypotheses, the value of .5 for $m$ produced a fit to the overall pattern of results that was as good as, or better than, any of the other values tested; with other values of $m$, the fit was improved for some conditions, but worse for others. In particular, values of $m<0$ produced a very poor fit to the present results because the decay functions, and hence the predicted changes across conditions, were far too extreme.

With $m=.5$, both equations generated values consistent with most trends observed in the present experiments. However, a few discrepancies are apparent. For example, the finding that ITI effects were smaller when the baseline delay was $0 \mathrm{sec}$ (Experiment 4) than when the delay was $10 \mathrm{sec}$ (Experiments 1 and 2) is particularly problematic for the relative-duration hypothesis. Since variations in the ITI change sample-to-background ratios to a greater extent when the delay is $0 \mathrm{sec}$ than when the delay is $10 \mathrm{sec}$, the relative-duration hypothesis predicts that systematic error tendencies arising from ITI variation should be more, rather than less, pronounced when the delay is 0 sec.

Both hypotheses also have problems with the tests in which the short ITI was combined with a long delay (i.e., the 5-sec ITI/20-sec delay trials of Experiment 3). Using either equation, this condition generates values that approximate baseline values. Therefore, the 5-sec ITI not only should have counteracted the choose-short tendency normally seen at the 20 -sec test delay, but also should have returned overall accuracy levels to near baseline levels. Instead, the birds were just as inaccurate in this condition as they were in the $90-\sec$ ITI/20-sec delay condition, albeit less biased toward choose-short errors.

The match of predictions from these models to the present results is not perfect, but it is close enough to warrant further investigation. Based on the present set of data, the temporal-summation hypothesis appears to provide a somewhat better fit. However, further tests are needed to clearly differentiate between these hypotheses and to determine whether either one provides a reasonable account of pigeons' memory for event duration in delayed matching tasks.

Although it is clear from the present set of results that pigeons' discrimination and retention of event durations is affected by the temporal context in which the events occur, many questions remain about this relationship. For example, how important is the similarity of stimulus conditions (e.g., a dark chamber) during the delay interval and ITI for the emergence of systematic errors in response to delay and ITI variation? How important is the constancy of the ITI and delay during initial training? According to the relative-duration hypothesis, for example, pigeons should use both the delay and ITI as part of a common background only to the extent that they are similar. In addition, the extent to which each has been stable during training should affect the likelihood that pigeons will be sensitive to variations in their duration during test sessions. Both the temporal-summation hypothesis and the relative-duration hypothesis also predict that the absolute value of the baseline ITI should be important for the magnitude of the choose-short effect produced by delay increases: The effect should be more pronounced in the context of a short ITI than in the context of a long ITI. Finally, the temporal summation hypothesis predicts that the presentation of an extremely long sample on one trial should increase the tendency to respond long on the next trial, even when reasonably lengthy ITIs are used. These and other predictions are currently under investigation.

\section{REFERENCES}

Church, R. M. (1980). Short-term memory for time intervals. Learning \& Motivation, 11, 208-219.

Fetterman, J. G. , \& Dreyfus, L. R. (1986). Pair comparisons of duration. Behavioural Processes, 12, 111-123. 
Fetterman, J. G., Stubbs, D. A., Dreyfus, L. R., Regan, L. M., \& BeRnsteIn, E. H. (1985, April). Discrimination of duration ratios. Paper presented at the meeting of the Eastern Psychological Association, Boston.

Killeen, P. R., \& Fetterman, J. G. (1988). A behavioral theory of timing. Psychological Review, 95, 274-285.

Kraemer, P. J., Mazmanian, D. S., \& Roberts, W. A. (1985). The choose-short effect in pigeon memory for event duration: Subjective shortening versus coding models. Animal Learning \& Behavior, 13, 349-354

MECK, W. H., \& ChURCh, R.. M. (1982). Discrimination of intertrial intervals in cross-modal transfer of duration. Bulletin of the Psychonomic Society, 19, 234-236.

Roberts, W. A., \& Kraemer, P. J. (1982). Some observations of the effects of intertrial interval and delay on delayed matching to sample in pigeons. Journal of Experimental Psychology: Animal Behavior Processes, 8, 342-353.

SANTI, A. (1984). The trial spacing effect in delayed matching-to-sample by pigeons is dependent upon the illumination condition during the intertrial interval. Canadian Journal of Psychology, 38, 154-165.

SPETCH, M. L. (1987). Systematic errors in pigeons' memory for event duration: Interaction between training and test delay. Animal Learning \& Behavior, 15, 1-5.

SPETCh, M. L., \& Sinha, S. S. (1988, November). Pigeons' memory for time: Evidence for retrospective processing. Paper presented at the meeting of the Psychonomic Society, Chicago.

SPETCH, M. L., \& WILKIE, D. M. (1982). A systematic bias in pigeons' memory for food and light durations. Behaviour Analysis Letters, 2 , 267-274

SPETCH, M. L., \& WILKIE, D. M. (1983). Subjective shortening: A model of pigeons' memory for event durations. Journal of Experimental Psychology: Animal Behavior Processes, 9, 14-30.

Staddon, J. E. R. (1984). Time and memory. Annals of the New York Academy of Sciences, 423, 322-334.

StuBBS, D. A. (1976). Scaling of stimulus duration by pigeons. Journal of the Experimental Analysis of Behavior, 26, 15-25.

Stubbs, D. A., Dreyfus, L. R., \& Fetterman, J. G. (1984). The perception of temporal events. In J. Gibbon \& L. Allan (Eds.), Timing and time perception. Annals of the New York Academy of Sciences, 423, 31-42.

WILKIE, D. M. (1984). Pigeons' spatial memory: IV. Effects of intertrial interval manipulations on delayed matching of key locations. Canadian Journal of Psychology, 38, 178-195.

WILKIE, D. M. (1988). Proactive effects in pigeons' timing behavior: Implications for an internal-clock model. Animal Leaming \& Behavior, $16,132-136$.

\section{APPENDIX}

\section{Relative Duration Hypothesis}

The ratio values generated by Equation 1,

$$
S=\left(t_{2}^{m}-t_{1}^{m}\right)_{s} /\left[\left(t_{2}^{m}-t_{1}^{m}\right)_{d}+\left(t_{2}^{m}-t_{1}^{m}\right)_{i}\right]
$$

(with $m=.5$ ), are shown below for each condition of Experiments 1,3 , and 4 . The values for baseline trials are underlined. The delay of $0 \mathrm{sec}$ was given a functional value of $1 \mathrm{sec}$ (assuming that it would take the bird at least $1 \mathrm{sec}$ to remove its head from the hopper and look at the response keys).

\section{Experiment 1}

\begin{tabular}{|c|c|c|c|c|c|c|c|c|c|c|}
\hline \multirow{3}{*}{$\begin{array}{l}\text { Delay } \\
\text { ITI }\end{array}$} & \multicolumn{5}{|c|}{ Delay Tests } & \multicolumn{5}{|c|}{ ITI Tests } \\
\hline & 0 & 5 & 10 & 15 & 20 & 10 & 10 & 10 & 10 & 10 \\
\hline & 45 & 45 & 45 & 45 & 45 & 5 & 15 & 45 & 75 & 90 \\
\hline Short & .12 & .06 & .04 & .03 & .03 & .08 & .06 & .04 & .03 & .03 \\
\hline Long & .37 & .22 & .16 & .13 & .11 & .29 & .23 & .16 & .13 & .12 \\
\hline
\end{tabular}

\section{Experiment 3}

\begin{tabular}{lrrrrr}
\hline Delay & 0 & 0 & 10 & 20 & 20 \\
ITI & 5 & 90 & 45 & 5 & 90 \\
Short & .35 & .08 & .04 & .04 & .02 \\
Long & 1.14 & .25 & $\underline{.16}$ & .17 & .08 \\
\hline
\end{tabular}

Experiment 4

\begin{tabular}{lrrrrrrr} 
& \multicolumn{3}{c}{ Delay Tests } & & \multicolumn{3}{c}{ ITI Tests } \\
\cline { 2 - 4 } Delay & 0 & 5 & 10 & & 0 & 0 & 0 \\
ITI & 45 & 45 & 45 & & 5 & 45 & 90 \\
Short & .12 & .06 & .04 & & .35 & $\underline{.12}$ & .08 \\
Long &. .37 & .22 & .16 & & 1.14 & $\underline{.37}$ & .25 \\
\hline
\end{tabular}

\section{Temporal Summation Hypothesis}

The values generated by Equation 2,

$$
S=\left(t_{2}^{m}-t_{1}^{m}\right)_{n}+\left(t_{2}^{m}-t_{1}^{m}\right)_{n-1}+\ldots\left(t_{2}^{m}-t_{1}^{m}\right)_{n-n},
$$

(with $m=.5$ ) are shown below for each condition of Experiments 1, 3, and 4. Again, the values for baseline trials are underlined.

To generate these values, we used only the current trial and the two preceding trials for our calculations, because samples from earlier trials add only trivial amounts to the aggregate sample. In addition, a number of specific assumptions had to be made. First, since food was used as the sample, it was assumed that both the sample and the reinforcer from previous trials could summate with the current sample value. Second, since the sample was sometimes $2 \mathrm{sec}$ and sometimes $8 \mathrm{sec}$, a mean sample duration of $5 \sec$ was used for Trials $n-1$ and $n-2$. Third, for determining the age of samples and reinforcers on Trials $n-1$ and $n-2$, we used the mean value of $10 \mathrm{sec}$ for the delay and $45 \mathrm{sec}$ for the ITI that preceded Trial $n-1$, even though the actual values varied around these means. Fourth, as with the previous calculations, we used a functional value of $1 \mathrm{sec}$ for the 0sec delay.

\begin{tabular}{|c|c|c|c|c|c|c|c|c|c|c|}
\hline \multirow{3}{*}{$\begin{array}{l}\text { Delay } \\
\text { ITI }\end{array}$} & \multicolumn{5}{|c|}{ Delay Tests } & \multicolumn{5}{|c|}{ ITI Tests } \\
\hline & 0 & 5 & 10 & 15 & 20 & 10 & 10 & 10 & 10 & 10 \\
\hline & 45 & 45 & 45 & 45 & 45 & 5 & 15 & 45 & 75 & 90 \\
\hline Short & 1.7 & 1.4 & $\underline{1.2}$ & 1.2 & 1.1 & 1.7 & 1.5 & 1.2 & 1.1 & 1.1 \\
\hline Long & 3.0 & 2.3 & 2.0 & 1.8 & 1.7 & 2.4 & 2.2 & $\underline{2.0}$ & 1.9 & 1.8 \\
\hline
\end{tabular}

\section{Experiment 1}

Experiment 3

\begin{tabular}{lrrrrr}
\hline Delay & 0 & 0 & 10 & 20 & 20 \\
ITI & 5 & 90 & 45 & 5 & 90 \\
Short & 2.4 & 1.5 & $\underline{1.2}$ & 1.4 & 1.0 \\
Long & 3.4 & 2.8 & $\underline{2.0}$ & 2.0 & 1.6 \\
\hline
\end{tabular}

Experiment 4

\begin{tabular}{lrrrrrrr}
\hline & \multicolumn{3}{c}{ Delay Tests } & & \multicolumn{3}{c}{ ITI Tests } \\
\cline { 2 - 4 } \cline { 6 - 8 } Delay & $\mathbf{0}$ & 5 & 10 & & 0 & 0 & 0 \\
ITI & 45 & 45 & 45 & & 5 & 45 & 90 \\
Short & $\underline{1.7}$ & 1.4 & 1.2 & & 2.4 & $\underline{1.7}$ & 1.5 \\
Long & $\underline{\mathbf{3 . 0}}$ & 2.3 & 2.0 & & 3.4 & $\underline{\underline{3.0}}$ & 2.8 \\
\hline
\end{tabular}

(Manuscript received February 26, 1988; revision accepted for publication August 18, 1988.) 